Acta Crystallographica Section A

Foundations of Crystallography

ISSN 0108-7673

Received 18 May 2004 Accepted 24 June 2004

(C) 2004 International Union of Crystallography Printed in Great Britain - all rights reserved

\section{Three-periodic nets and tilings: minimal nets}

\author{
Charlotte Bonneau, ${ }^{a}$ Olaf Delgado-Friedrichs, ${ }^{b}$ Michael $\mathrm{O}^{\prime} \mathrm{Keeffe}^{\mathrm{a} *}$ and \\ Omar M. Yaghi ${ }^{\mathrm{C}}$
}

\begin{abstract}
${ }^{\mathbf{a}}$ Department of Chemistry, Arizona State University, Tempe, AZ 85287, USA, ${ }^{\mathbf{b}}$ Department of Computer Science, University of Tübingen, D-72072 Tübingen, Germany, and ${ }^{\mathbf{c}}$ Department of Chemistry, University of Michigan, Ann Arbor, MI 48109, USA. Correspondence e-mail: mokeeffe@asu.edu
\end{abstract}

The 15 3-periodic minimal nets of Beukemann \& Klee [Z. Kristallogr. (1992), 201, 37-51] have been examined. Seven have collisions in barycentric coordinates and are self-entangled. The other eight have natural tilings. Five of these tilings are self-dual and the nets are the labyrinth nets of the $P, G, D, H$ and CLP minimal surfaces of genus 3. Twelve ways have been found for subdividing a cube into smaller tiles without introducing new vertices. Duals of such tilings with one vertex in the primitive cell have nets that are one of the minimal nets. Minimal nets without collisions are uniform.

\section{Introduction}

This paper is the third in a series on the taxonomy of 3-periodic tilings and nets. The first two (Delgado-Friedrichs et al., $2003 a, b)$ papers dealt with vertex- and edge-transitive nets. In this contribution, we discuss the minimal nets defined and enumerated by Beukemann \& Klee (1992).

We first give a brief informal summary of definitions. In this work, a tile is a closed figure made of vertices, edges and faces such that at least two edges meet at each vertex, and two faces meet at each edge. A tiling is a filling of space by tiles sharing faces. Each tiling has a unique dual obtained by placing a vertex in the original tile and connecting these new vertices by edges passing through the faces of the old tiles. The new tiles enclose the original vertices and the original tiling is the dual of the dual.

We say that each tiling carries a net that is composed of the set of its vertices and edges. Although the net of a tiling is unique, the converse is not true; one can derive a new tiling from a given tiling with the same net either by fusing pairs of tiles (eliminating faces) or by dissecting tiles with more than four vertices (adding faces). However, we have proposed that there is often a unique natural tiling associated with a net. This natural tiling has the property that the tiles are the smallest possible $(a)$ that preserve the full combinatorial symmetry of the net and $(b)$ whose faces are all strong rings (i.e. rings that are not the sum of smaller rings).

Often the dual of a natural tiling is also a natural tiling, and then we refer to the pairs of nets as natural duals. If the tiling and its dual are identical, they are self-dual and, if they are natural tilings, we say the nets are naturally self-dual (or informally just self-dual). ${ }^{1}$

\footnotetext{
$\mathbf{1}$ Thus the term 'dual net' is to be construed to mean the net of the tiling dual to the tiling of the original net. As a net has infinitely many tilings (provided that at least one exists), there are infinitely many duals.
}

The transitivity pqrs of a tiling signifies that there are $p$ kinds of vertex, $q$ kinds of edge, $r$ kinds of face and $s$ kinds of tile. The dual of a tiling with transitivity pqrs has transitivity srqp.

If the edges of a repeat unit (i.e. primitive unit-cell contents) of a 3-periodic net that connect to a vertex in the $u v w$ unit cell are joined to their partners connecting to corresponding vertices in the $\bar{u} \bar{v} \bar{w}$ unit cell, one obtains a quotient graph (Chung et al., 1984) that may contain loops and/ or have more than one edge connecting the same pair of vertices (see Fig. 1). If we now imagine the edges to be inflated to rods of finite circumference, the quotient graph will correspond to a handlebody of genus $g$. We find it convenient to refer to the genus of a net, defined in this way. If the $v$ vertices of the quotient graph are linked by the minimal number $(v-1)$ of edges to make a connected graph, one obtains a spanning tree of the graph. The number of edges required to complete the graph is the cyclomatic number, which is equal to $g$. If the total number of edges in the quotient graph is $e$ then the cyclomatic number is $g=e-(v-1)=$ $1+e-v$.

For 3-periodic tilings, the number of vertices, edges, faces $(f)$ and tiles $(t)$ in the repeat unit are related by $e-v=f-t$ (Coxeter, 1973). As the dual tiling has $f$ edges and $t$ vertices, it is a property of three-dimensional tilings that the genus of the net of the dual tiling $(1+f-t)$ is the same as the genus $(1+e-$ $v$ ) of the net of the original tiling (notice that this is not true in two dimensions).

The concept of a minimal net was introduced by Beukemann \& Klee (1992); it has the minimum possible number of vertices and edges in the repeat unit. For an $n$-periodic net, the genus of the minimal net is $n$. Beukemann \& Klee (1992) showed that to enumerate the minimal nets it is sufficient to enumerate all the connected graphs of cyclomatic number $n$. For $n=3$, the number of such graphs is 15 . 
Table 1

The minimal nets without collisions.

'BK' refers to the symbol of Beukemann \& Klee (1992), 'Coord.' refers to the coordination number(s) of the vertices and 'Trans.' is the transitivity described in the text. In the vertex symbol (O'Keeffe \& Hyde, 1996), $N_{n}$ for each angle means that $n N$-rings meet at that angle; '*' signifies that there are no rings contained in that angle.

\begin{tabular}{|c|c|c|c|c|c|c|}
\hline Net & BK & Symmetry & Coord. & Trans. & Dual & Vertex symbols \\
\hline pcu & $1(6) 1$ & $P m \overline{3} m$ & 6 & 1111 & self & 4.4.4.4.4.4.4.4.4.4.4.4.*.*.* \\
\hline hms & $2(3,5) 1$ & $P \overline{6} m 2$ & $5 ; 3$ & 2222 & self & $6 \cdot 6 \cdot 6 \cdot 6_{2} \cdot 6_{2} \cdot 6_{2} \cdot 6_{2} \cdot 6_{2} \cdot 6_{2} \cdot * ; 6_{3} \cdot 6_{3} \cdot 6_{3}$ \\
\hline dia & $2(4) 1$ & $F d \overline{3} m$ & 4 & 1111 & self & $6_{2} \cdot 6_{2} \cdot 6_{2} \cdot 6_{2} \cdot 6_{2} \cdot 6_{2}$ \\
\hline cds & 2(4) 2 & $P 4_{2} / m m c$ & 4 & 1221 & self & $6 \cdot 6 \cdot 6 \cdot 6 \cdot 6_{2} \cdot *$ \\
\hline tfa & $3\left(3^{2}, 4\right) 1$ & $I \overline{4} m 2$ & $4 ; 3$ & 2211 & dia & $8_{2} \cdot 8_{2} \cdot 8_{3} \cdot 8_{3} \cdot 8_{3} \cdot 8_{3} ; 8_{4} \cdot 8_{4} \cdot 8_{4}$ \\
\hline tfc & $3\left(3^{2}, 4\right) 3$ & $\mathrm{Cmmm}$ & $4 ; 3$ & 2321 & pcu & $8_{2} \cdot 8_{2} \cdot 8_{2} \cdot 8_{2} \cdot 8_{2} \cdot * ; 8 \cdot 8_{3} \cdot 8_{3}$ \\
\hline srs & $4(3) 1$ & $I 4,32$ & 3 & 1111 & self & $10_{5} \cdot 10_{5} \cdot 10_{5}$ \\
\hline ths & $4(3) 2$ & $I 4_{1} /$ amd & 3 & 1211 & dia & $10_{2} \cdot 10_{2} \cdot 10_{4}$ \\
\hline
\end{tabular}

\section{Nets with collisions and self-catenation}

Nets with catenated rings occasionally occur in crystal chemistry (O'Keeffe, 1991; O'Keeffe et al., 2000). Such rings cannot be faces of tiles, and in general there may be no natural tiling associated with the net.

In analyzing the topology of nets, we find it convenient to work with barycentric coordinates (Delgado-Friedrichs \& O'Keeffe, 2003). However, there are certain kinds of net for which two or more vertices have the same coordinates (collide). We have not encountered such behavior in nets of interest to crystal chemistry. Fig. 1(b) illustrates such a net (one of the minimal nets of Beukemann \& Klee). ${ }^{2}$ Consider the red vertex placed, without loss of generality, at $(0,0,0)$ (so the eight red vertices shown are at the corners of a unit cell) and the green vertex at $(x, y, z)$. Take the $z$ direction to be up the page. Then the neighbors of the red vertex are at $(1,0,0)$, $(-1,0,0),(0,1,0),(0,-1,0)$ and $(x, y, z)$. For the coordinates to be barycentric these numbers must add up to $(0,0,0)$, i.e. $x=y=z=0$ and the red and green vertices collide.

Of the 15 minimal 3-periodic nets of Beukemann \& Klee (1992), we find that seven have collisions and that these are all self-catenated. They all have 3-coordinated vertices with the vertex on a single loop in the quotient graph [cf. the green vertex in Fig. 1(d)]. We do not consider these structures any further.

\section{Minimal nets without collisions}

The eight minimal nets without collisions are listed in Table 1 and tiles of their natural tilings are shown in Fig. 2. The nets pcu, dia and srs with transitivity 1111 are regular nets and have been so described before (Delgado-Friedrichs et al., 2003a). As they are naturally self-dual, it is common to find crystal structures based on two or more of a given kind of net intergrown (Batten \& Robson, 1998). The nets correspond to the labyrinth systems of the periodic minimal surfaces (PMSs) $P$, $D$ and $G$, respectively (for desciptions of PMSs see e.g.

\footnotetext{
2 This net is labeled 2(3,4)2 by Beukemann \& Klee (1992) in their Fig. 1(b); this is clearly a misprint for $2(3,5) 2$ as given in the caption for our Fig. 1
}

Karchner, 1989; Hyde \& Ramsden, 2000). The net cds $\left(\mathrm{CdSO}_{4}\right)$ with transitivity 1221 is also self-dual and crystal structures with intergrown nets with this topology have been described (Delgado-Friedrichs et al., 2003c). This net is the labyrinth system of the CLP PMS. The last naturally self-dual net is that labeled hms with transitivity 2222; it is the labyrinth graph of the $H$ PMS. Examples of crystal structures based on pairs of these nets intergrown have been given by Batten \& Robson (1998, §4.4.2).

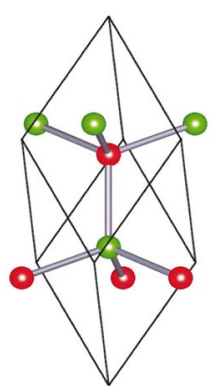

(a)

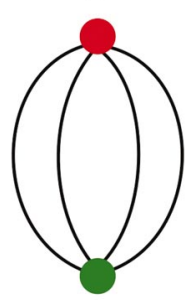

(c)

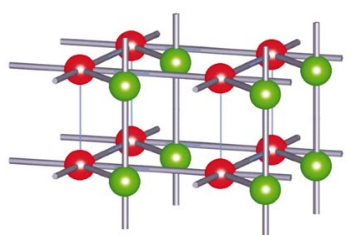

(b)

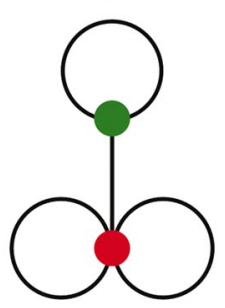

(d)
Figure 1

(a) A fragment of the dia structure. (b) A minimal net $[2(3,5) 2$ of Beukemann \& Klee (1992)]. (c) The quotient graph of dia. (d) The quotient graph of the net in $(b)$.
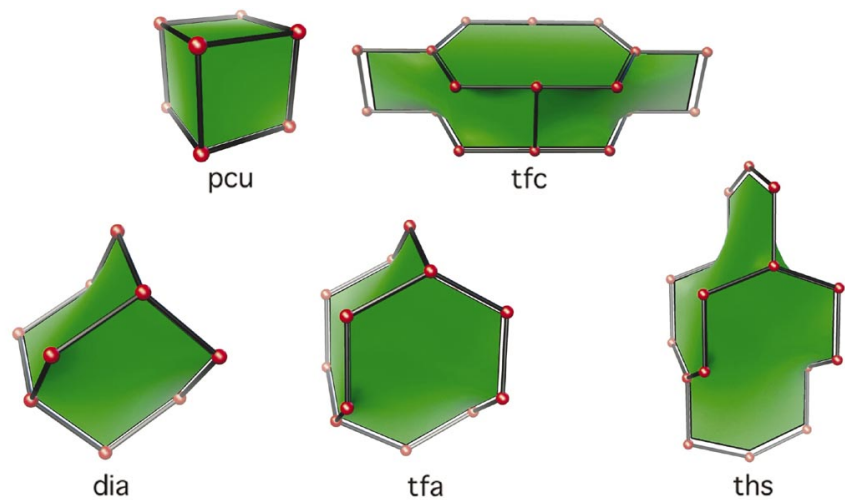

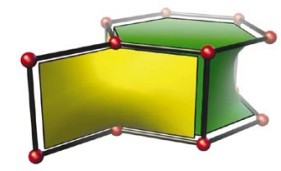

$\mathrm{hms}$
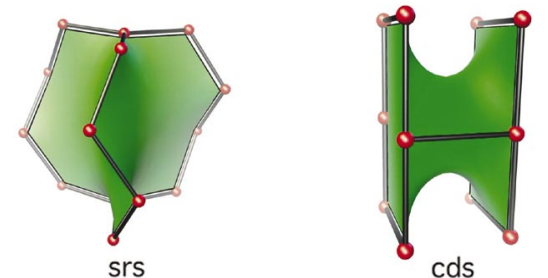

Figure 2

Natural tiles of the minimal nets without collisions ( $c f$. Table 1). 
The one-to-one correspondence of the five naturally selfdual minimal nets and the labyrinth systems of the five minimal surfaces of genus 3 might have been expected [for the genera of minimal surfaces see Fischer \& Koch (1989); in that work the $G$ surface is labeled $Y^{*}$ ].

The net labeled ths is familiar as the net of the Si atoms in $\mathrm{ThSi}_{2}$. The dual of the natural tiling is a tiling that carries the dia net, but this second tiling is not a natural tiling; indeed, as shown in Fig. 3(a), the dual tiling is composed of trihedral tiles, which are obtained by dividing the dia natural tile (Fig. 2) into halves. The close relationship between ths and dia means that structures often occur with two or more intergrown ths nets (Batten \& Robson, 1998; O'Keeffe et al., 2000) although not all edges (i.e. not those parallel to the tetragonal $c$ axis) of one net penetrate rings of a second. Note that we can construct a self-dual tiling of ths by bisecting the natural tiling as shown in Fig. 3(b). One of the faces of the new tiles has 12 edges and is not a ring; from the figure it should be clear that the face is the sum of two 10-rings (the other two faces of the tile). The transitivity of this new tiling is 1221 .

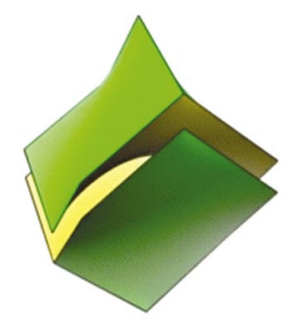

(a)

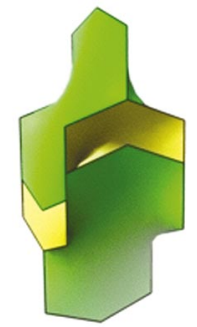

(b)
Figure 3

(a) The tiling dual to the natural tiling of ths. Compare with the natural tile of dia (Fig. 2). (b) A self-dual tiling of ths. Compare with the natural tile of ths (Fig. 2).

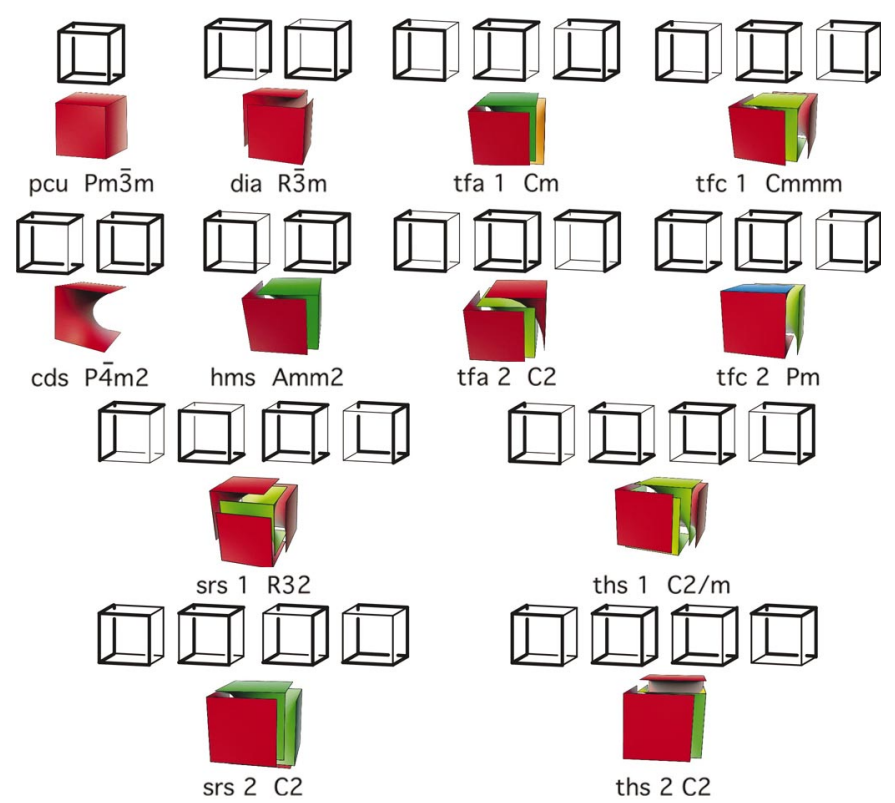

Figure 4

Tilings derived by dissecting a cube. The derived minimal net of the dual tiling is indicated under each tiling ( $c f$. Table 1).
The 3,4-coordinated net labeled tfa is intermediate between dia and ths, and again the net of the dual to the natural tiling is dia. The second 3,4-coordinated net is labeled tff; the net of the dual of its natural tiling is pcu. These nets with their lower symmetry and mixed coordination are of lesser importance in crystal chemistry.

For each of these nets, the shortest ring at each angle is the same size and, in fact, is equal to $2 v+2$. Wells (1977) attached special interest to such nets, which he called uniform. Notice however that the cds net also has 8-rings that serve as faces of natural tiles (six of these 8-rings meet in each ' $90^{\circ}$ ' angle) and which are therefore part of the set of essential rings (DelgadoFriedrichs et al., 2003a).

\section{Minimal tilings of the primitive cubic net (pcu)}

A tiling by cubes carries the pcu minimal net. If we subdivide the cube into smaller tiles, keeping the cube faces intact, we will have tilings that carry the same net (for convenience we call these minimal tilings). The duals of these tilings must be tilings that carry a minimal net, so it is of interest to see whether the nets generated in this way correspond to the minimal nets described above. Fig. 4 shows 12 different such dissections of a cube (including the case where the cube is left intact). The duals of the tilings do indeed generate the eight minimal nets of this paper; four are generated twice.

This result suggests that one could systematically enumerate the dissections of a cube starting from the quotient graphs of the minimal nets. These graphs consist conceptually of two parts: a spanning tree, and three extra directed edges (which may be loops) labeled $x, y$ and $z$. Now let us label opposite faces of a cube $\pm x, \pm y$ and $\pm z$, and insert a spanning tree of a minimal quotient graph inside. The edges of the spanning tree go through faces of tiles in the dissected cube and the labeled edges go to the corresponding labeled faces of the cube. Thus for each vertex we have a dual tile arising from the one or two incident edges of the spanning tree and the directed edges. Fig. 5 shows the quotient graphs of the eight minimal nets without collisions. It may be seen that for four of the nets the spanning tree may be chosen in two distinct ways. There is a one-to-one correspondence between these 12

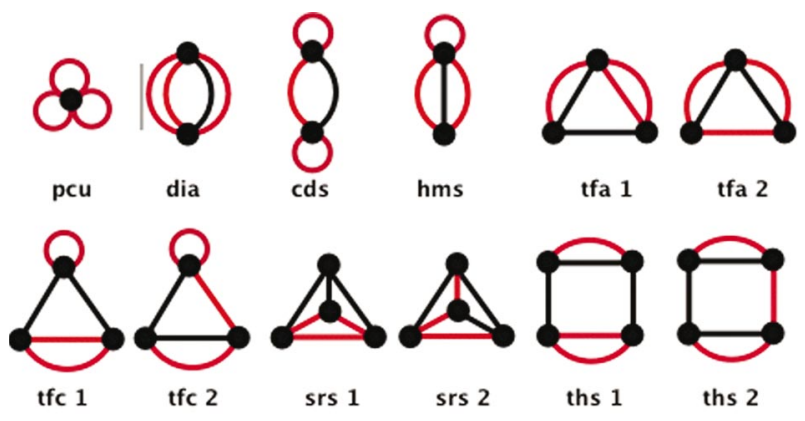

Figure 5

The quotient graphs of the minimal nets. Black edges define a spanning tree, and the three red edges are labeled $x, y$ and $z$. The graphs correspond to the tilings of Fig. 4. 
graphs and the 12 dissections of the cube, so we believe the enumeration is complete.

This approach to minimal nets shows why there are no tilings associated with the minimal nets with collisions. The quotient graphs for these nets all have at least one trivalent vertex with an incident loop. The dual tile associated with such a vertex must have three faces, of which two are opposite faces of a cube (associated with the incoming and outgoing part of the incident loop). Clearly it is impossible to have a tile with three faces in which two faces have no common edge.

The results above also suggest that dissections of basic tilings might be a fruitful route to generating nets that can be described as tilings. A difficulty in attempting to enumerate nets from quotient graphs is the explosive growth of number of topologies with genus. Thus for 3-coordinated nets in progressing from genus 3 with five possibilities to genus 4 , even with restrictive assumptions, 269 possibilities have been found (Bader et al., 1997); the majority of these are not plausible topologies to serve as the basis for crystal structures.

\section{Concluding remarks}

Five of the eight nets described in this work are naturally selfdual, yet among all known nets this is a very rare property; indeed, we believe that the only vertex-transitive naturally self-dual nets are the four (pcu, dia, srs and cds) described here. We believe that, in most cases of crystal structures based on interpenetrating nets, the net topology will be derived from one of these four basic nets. Examples of interpenetrating structures based on nets derived from the cds net have been given by Delgado-Friedrichs et al. (2003c). A subsequent paper in this series will be devoted to the nets of other selfdual tilings.

This work was supported by the US National Science Foundation (grant No. DMR 0103036).

\section{References}

Bader, M., Klee, W. E. \& Thimm, G. (1997). Z. Kristallogr. 212, 553-558.

Batten, S. R. \& Robson, R. (1998). Angew. Chem. Int. Ed. 37, 1461-1494.

Beukemann, A. \& Klee, W. A. (1992). Z. Kristallogr. 201, 37-51.

Chung, S. J., Hahn, Th. \& Klee, W. E. (1984). Acta Cryst. A40, 42-50.

Coxeter, H. S. M. (1973). Regular Polytopes, 3rd ed., §9.8. New York: Dover Publications.

Delgado-Friedrichs, O. \& O'Keeffe, M. (2003). Acta Cryst. A59, 351-360.

Delgado-Friedrichs, O., O'Keeffe, M. \& Yaghi, O. M. (2003a). Acta Cryst. A59, 22-27.

Delgado-Friedrichs, O., O'Keeffe, M. \& Yaghi, O. M. (2003b). Acta Cryst. A59, 515-525.

Delgado-Friedrichs, O., O'Keeffe, M. \& Yaghi, O. M. (2003c). Solid State Sci. 5, 73-78.

Fischer, W. \& Koch, E. (1989). Acta Cryst. A45, 726-732.

Hyde, S. T. \& Ramsden, S. (2000). Chemical Topology: Applications and Techniques, edited by D. Bonchev \& D. H. Rouvray, ch. 2. Amsterdam: Gordon and Breach.

Karchner, H. (1989). Manuscripta Math. 64, 291-357.

O'Keeffe, M. (1991). Z. Kristallogr. 196, 21-37.

O'Keeffe, M., Eddaoudi, M., Li, H., Reineke, T. M. \& Yaghi, O. M. (2000). J. Solid State Chem. 152, 2-20.

O'Keeffe. M. \& Hyde, B. G. (1996). Crystal Structures: Patterns and Symmetry. Washington, DC: Mineralogical Society of America.

Wells, A. F. (1977). Three-Dimensional Nets and Polyhedra. New York: Wiley. 